\title{
Prolonged anesthesia alters brain synaptic architecture
}

\author{
Michael Wenzel, Alexander Leunig, Shuting Han, Darcy S. Peterka and Rafael Yuste \\ Neurotechnology Center, Department of Biological Sciences, Columbia University, New York, \\ NY 10027, USA
}

Correspondence to:

Michael Wenzel

902 NWC Building 550 West 120 Street, Box 4822, New York, NY, 10027.

Email: michaelwenzel2946@gmail.com

Phone: +1 2128545023

M.W.'s present address: Department of Epileptology, University of Bonn, 53127 Bonn, Germany.

D.S.P.'s present address: Zuckerman Mind Brain Behavior Institute, Columbia University, New York, NY 10027, USA

Conflict of Interest: The authors declare no competing financial interests 
SUMMARY

2 Prolonged medically-induced coma (pMIC), a procedure performed in millions of

3 patients worldwide, leads to cognitive impairment, yet the underlying brain mechanism

4 remains unknown. No experimental studies of medically-induced coma (MIC)

5 exceeding $\sim 6$ hours exist. For MIC of less than 6 hours, studies in developing rodents

6 have documented transient changes of cortical synapse formation. However, in

7 adulthood, cortical synapses are thought to become stabilized. Here, we establish pMIC

8 (up to $24 \mathrm{hrs}$ ) in adolescent and mature mice, and combine repeated behavioral object

9 recognition assessments with longitudinal two-photon imaging of cortical synapses. We

10 find that pMIC affects cognitive function, and is associated with enhanced synaptic

11 turnover, generated by enhanced synapse formation during pMIC, while the post-

12 anesthetic period is dominated by synaptic loss. These results carry profound

13 implications for intensive medical care, as they point out at significant structural side

14 effects of pMIC on cortical brain synaptic architecture across age levels.

16 In humans, cognitive impairment due to prolonged medically induced coma (pMIC)

17 represents an enormous clinical and socio-economic burden affecting millions of

18 patients worldwide ${ }^{1-5}$. In adulthood, clinical trials have proven that intensive care unit

19 (ICU) survivors frequently suffer from lasting cognitive impairment ${ }^{1,6}$, yet its

20 pathophysiology and neuromorphopathological underpinnings have remained elusive. In

21 early life (childhood and early adolescence), when the brain is highly plastic, MIC (max.

226 hrs tested in rodents to date) has been shown to result in synaptic changes and long-

23 term cognitive impairment ${ }^{7-9}$. However, basic animal research suggests that during later 
24 adolescence and adulthood, dendrites and dendritic spines become stabilized under

25 physiological conditions ${ }^{10-12}$, and short-term $\mathrm{MIC}^{13}$. Whether this notion holds true for

26 pMIC, is unknown.

28 There has been a paucity of experimental studies of structural and functional

29 consequences of pMIC, so our first objective was to create a robust platform for 30 measurement in mice. We combined a mobile anesthesia setup (Fig. 1a), chronic two-

31 photon imaging of cortical synapses ${ }^{14}$ (Fig. 1b) through a reinforced thin skull window ${ }^{15}$

32 (Fig. 1b), and repeated behavioral assessment ${ }^{16}$ (Fig. 1c) into an integrated

33 experimental framework (Fig. 1d, please see methods). Two imaging sessions were

34 carried out to determine baseline 3-day ('d') synaptic turnover (d1, d4). Immediately 35 after d4 imaging, pMIC was initiated. To document synaptic changes across pMIC, a 36 third imaging session was carried out immediately upon pMIC discontinuation (d5).

37 Three days post pMIC, mice were imaged once again to determine post-anesthetic 3-

38 day synaptic turnover (d8). Additionally, a novel object recognition test ${ }^{16}$ was performed

39 during baseline conditions (d4), and repeated after recovery from pMIC (d7, fig. 1c).

41 We used the inhalatory anesthetic isoflurane due to its fast kinetics (order of minutes)

42 and thus maximal adjustability of anesthetic depth ${ }^{17}$. We tried to avoid an $\mathrm{FiO}_{2}$ (fraction

43 of inspired oxygen) $>50 \%$ throughout pMIC, as high $\mathrm{FiO}_{2}$ values have been associated

44 with increased mortality in human intensive care ${ }^{18}$. Vital parameters (peripheral $\mathrm{O}_{2}$

45 saturation, heart rate, breath rate, pulse distention, breath distention) were closely

46 monitored using an infrared thigh sensor (Fig. 1e). When developing the experimental 
47 protocol, we successfully performed extended anesthesia in mice for up to 40 hours, yet

48 for reasons of experimental feasibility (continuous presence of an experimenter

49 required), we carried out pMIC on 12 adult mice (age: $7.7 \pm 1.5$ months s.e.m.) with a 50 mean duration of 18 hours (17.96 \pm 0.74 s.e.m.). Cardiorespiratory instability, or

51 maximum pMIC duration of 24 hours were chosen as primary pMIC discontinuation

52 criteria. Fluid and nutritional supply was ensured by administration of glucose solution

53 (1\%, in PBS) via a subcutaneous line at $0.1-0.2 \mathrm{ml} / \mathrm{hr}$ (total volume $3.46 \pm 0.35 \mathrm{ml}$

54 s.e.m.). Under steady state isoflurane concentrations of $0.5-1.5 \%$ ppa, mice displayed

55 continuously high peripheral oxygen saturation (96.96 $\pm 0.32 \%$ s.e.m.), heart rates

56 between $350-500$ beats per minute (435 \pm 14 s.e.m.), breath rates of $50-80$ excursions

57 per minute $(65 \pm 3$ s.e.m.), and a pulse/breath distension ratio above 1 (1.75 \pm 0.16

58 s.e.m.) (Fig. 1e-f). Sufficient oxygenation (>90\%) was achieved with little variance

59 across a wide range of heart and breath rates (Fig. 1e-g). Relative variance of heart and

60 breath rate was much greater than $\mathrm{O}_{2}$ saturation. While heart and breath rate co-varied

61 over time (Fig. 1e), breath rate showed the largest variance of all monitored vital

62 parameters (Fig. 1g), and most accurately clinically reflected anesthetic depth. Upon

63 pMIC discontinuation, mice usually rapidly displayed an acceleration of heart and breath

64 rate, and soon reacted to tactile stimulation. We defined the period between isoflurane

65 discontinuation and first appearance of the righting reflex as the wake-up time (9.65 \pm

661.41 min s.e.m.). After waking up, all studied mice soon showed apparently normal

67 movement and body coordination as well as typical grooming and feeding behavior. To

68 evaluate whether pMIC results in basic behavioral changes, mice were repeatedly (prior

69 and post pMIC) subjected to a novel object recognition test (,NOR', fig. 1c). In line with 
70 previous literature ${ }^{16}$, mice spent significantly more time exploring novel objects post

71 familiarization during baseline conditions (Fig. 1h, left). However, this effect was

72 abolished post recovery (>24hrs) from pMIC (Fig. 1h, right). Importantly, this effect

73 could not be explained by a simple post-anesthetic reduction of navigation through the

74 maze, as pre- and post-anesthetic total distances travelled during both familiarization

75 and NOR were similar (Fig. 1i).

77 Next, we set out to study whether pMIC affects synaptic architecture. To this end, we

78 carried out repeated structural two-photon imaging of YFP-labeled dendrites and

79 synapses (up to $\sim 150 \mu \mathrm{m}$ beneath the pial surface) of layer 5 pyramidal neurons ${ }^{14}$ in 6 of

80 the 12 adult mice undergoing pMIC (Fig. 2a). We chose a minimally invasive approach,

81 using a reinforced chronic cranial window over the thinned skull to minimize possible

82 effects from invasive surgery. However, this reduces overall imaging fidelity, and we

83 were not able to consistently distinguish between filopodia and dendritic spines across

84 all imaging sessions in all mice. Therefore, we chose to focus on clearly visible synaptic

85 protrusions, regardless of morphological classification. In 6 mice, we assessed a total of

861254 synaptic protrusions (209 \pm 40 s.e.m.) on 238 dendritic compartments (40 \pm 9

87 s.e.m.). We noticed that across all anesthetized mice and imaging sessions, dendrites

88 remained stable (Fig. 2b-c). However, pMIC-associated synapse dynamics showed

89 considerable alterations (Fig. 2d). In comparison to the 1-day total synapse turnover in

90 adult controls ( $n=6$, total synapse count: 1113 [185.5 \pm 50 s.e.m.]), pMIC-associated

91 synapse turnover was more than doubled (Fig. 2e). Both synaptic gain and loss were

92 significantly enhanced during pMIC, with the gain of synapses outperforming their loss 
93 (Fig. 2f). When comparing pre- and post-anesthetic 3-day synaptic turnover, turnover

94 remained significantly enhanced after pMIC (Fig. 2g). In contrast to pMIC itself, the

95 post-anesthetic period was dominated by synaptic loss (Fig. 2h), while both post-

96 anesthetic synaptic gain and loss remained non-significantly increased. Intriguingly, a

97 substantial fraction of synapses gained during pMIC remained stable across the 3-day

98 post-anesthetic period (42.69 $\pm 10.87 \%$ s.e.m. [total gain: 59 synapses in $\mathrm{n}=6$ mice]).

99

100 Next, we sought to put our findings in adult mice in developmental context by examining 101 younger animals ( PND 30). Clinical studies, and animal research suggest that shorter 102 MIC periods (max. 6 hrs tested to date) at young age ( $\leq 1$ month in rodents) may be 103 associated with long-term cognitive impairment ${ }^{9}$, and increased synapse formation with 104 an age-dependently decreasing effect size (no MIC effect on dendritic spines at age >1 105 month) $)^{7,8,13}$.

106

107 Similarly to adult mice, pMIC in young mice $(n=4$, pMIC duration $=18.5 \pm 1.26 \mathrm{hrs}$ 108 s.e.m.) was associated with a significantly enhanced 1-day synapse turnover (Fig. 2i-j, $109 \mathrm{n}=4$ mice, total synapse count: 284 [71 \pm 12 s.e.m.]). In line with previous results on 110 immature synapses in 1 month old mice (isoflurane for $4 \mathrm{hrs})^{13}$, synaptic loss was 111 significantly reduced during pMIC compared to controls (Fig. $2 \mathrm{k}, \mathrm{n}=5$ mice, total 112 synapse count: 587 [117.4 \pm 15 s.e.m.]). At the same time, consistent with our results

113 for pMIC in adult mice, pMIC-associated synapse formation was strongly increased 114 resulting in a pronounced net-positive synaptic turnover (Fig. $2 \mathrm{k}$ ). When comparing the 115 pre- and post-anesthetic 3-day synaptic turnover, total turnover remained unchanged 
116 (Fig. 2I). Yet, in contrast with adult mice, synaptic formation post pMIC in younger mice

117 was significantly reduced, and synapse elimination increased, resulting in a net-

118 negative synaptic turnover (Fig. 2m). Again, a substantial fraction of synapses gained

119 during pMIC remained stable during the post-anesthetic period $(50.57 \pm 9.09 \%$ s.e.m.

120 [total gain: 36 synapses in $n=4$ mice]).

122 Our data demonstrate that prolonged general anesthesia alters object recognition and

123 cortical synaptic architecture in both young and adult mice. With isoflurane, our findings

124 point towards net-positive synapse turnover during pMIC, followed by a net-negative

125 synapse turnover during the post-anesthetic period. Across all examined ages, a high

126 fraction of newly formed spines during pMIC displayed unexpected post-anesthetic

127 stability suggesting that they may form persistent synaptic connections. Given the

128 fundamental relevance of synaptic connections and experience-driven synaptic

129 plasticity for cognitive function and memory ${ }^{19-21}$, our observed alterations in pMIC-

130 associated synaptic dynamics represent a possible factor contributing to cognitive

131 deficits following general anesthesia. The mechanisms underlying the observed

132 alterations remain unclear, yet likely play out across multiple anatomical scales ${ }^{22}$. On

133 the molecular level, anesthetics target ion channels ${ }^{23}$. On the organelle level,

134 anesthetics e.g. exert effects on a cell's cytoskeleton ${ }^{24}$. Ultimately, circuit level

135 alterations driven by anesthetics may play a main role in the pMIC-associated synaptic

136 plasticity observed here, since the prolonged pharmacological inactivation profoundly

137 disrupts neural circuits that under physiological conditions dynamically adapt at the 
138 synaptic level with changing intrinsic (e.g. circadian protein expression) ${ }^{25}$, and extrinsic

139 environmental parameters (e.g. novel sensory experience) across age ${ }^{21}$.

141 Our findings carry profound implications for medical care, as they point out at significant

142 structural and functional side effects of pMIC. To date, no standard approach exists to

143 prevent cognitive side effects or alterations of brain architecture during pMIC. A better

144 understanding of pMIC-related effects on cortical synapses, especially in the context of

145 different widely used anesthetics, could allow for more individually tailored anesthetic

146 regimens, and foster research on adjuvant therapeutic strategies. Such research may

147 pave the way towards methods to improve cognitive outcomes of ICU survivors, and

148 thus and help reduce the socioeconomic consequences of prolonged medically induced

149 coma.

151 Acknowledgments: We thank Yuste Lab members for useful comments on this project,

152 and H.W. Pfister for clinical supervision. Supported by the NEI (R01EY011787) and

153 NIMH (R01MH115900). This material is based upon work supported by, or in part by,

154 the U. S. Army Research Laboratory and the U. S. Army Research Office under contract 155 number W911NF-12-1-0594 (MURI). The authors have no competing financial interests

156 to declare. M.W. conceived the project, and designed the experimental framework.

157 M.W., D.S.P., and A.L. established the anesthesia setup. M.W. and A.L. performed the

158 experiments. M.W. (clinical data/vital parameters, structural imaging, behavioral

159 assessment), A.L. (clinical data/vital parameters, structural imaging, behavioral

160 assessment), D.S.P. (structural imaging), and S.H. (behavioral assessment) analyzed 
161 the data. S.H. provided custom code (MATLAB, MathWorks) for automated tracking of

162 mouse exploratory behavior. M.W. wrote the paper. All authors discussed results, and

163 edited the paper. R.Y. assembled and directed the team and secured funding, and

164 resources. R.Y. is an Ikerbasque Research Professor at the Donostia International

165 Physics Center.

Methods

General information. All experiments were carried out in compliance with the Columbia

170 University institutional animal care guidelines. We used B6.Cg-Tg(Thy1-YFP)HJrs/J

171 mice $^{14}$ (Jackson Laboratories; RRID: IMSR_JAX:003782), where cortical layer 5

172 pyramidal neurons are preferentially labeled. For experiments, postnatal age of mice

173 ranged from 1 to 12 months (two age groups: p30-35, or p120-360). Mice were housed

174 at a 12 hour light/dark cycle, and food and water was provided ad libitum.

176 Surgical procedures. For repeated two-photon imaging of cortical synapses, a

177 reinforced thin skull chronic cranial window was established as described ${ }^{15}$. We chose a

178 „thin skull“ approach to minimize potential craniotomy-related changes of synaptic 179 plasticity that have been reported previously ${ }^{26}$. In brief, mice were anaesthesized with

180 isoflurane (initial dose $2-3 \%$ partial pressure in air, then reduction to $1-1.5 \%$ ). Prior to 181 surgery, all mice received carprofen (s.c.), enrofloxacin (s.c.), and dexamethasone 182 (i.m.). Throughout surgical procedures, proper anesthetic depth was checked 183 intermittently by toe, and tail pinching. Under sterile conditions, a small flap of skin 184 above the skull was removed and a titanium head plate with a central foramen $(7 \times 7 \mathrm{~mm})$ 
185 was fixed on the skull using dental cement. Then, a dental microdrill was used at low

186 rotation speed to carefully thin a small region (usually $<1 \mathrm{~mm}$ in diameter) over the left

187 somatosensory cortex. To prevent the skull and brain tissue from heating up by the

188 drilling process, drilling and wetting with sterile artificial cereberospinal fluid (ACSF)

189 were alternated frequently. At a thickness of around $50 \mu \mathrm{m}$, the skull typically began to

190 bend upon gentle pressure and the intracranial vasculature became clearly visible under

191 the moistened skull. In addition, small air bubbles within the thin spongioform part of the

192 thinned skull appeared when ACSF was applied. From here on, the skull was further

193 thinned at an even lower drilling rotation speed and with minimal vertical pressure, down

194 to a thickness of $10-20 \mu \mathrm{m}$. When thin enough, the small air enclosures in the skull no

195 longer appeared upon ACSF application.

197 At this point, the skull was allowed to dry completely. Once dry, a small drop of 198 cyanoacrylate glue was applied onto the thinned skull. Immediately following the 199 application of the glue, a thin glass cover slip (3mm in diameter, No. 0, Warner 200 Instruments) was lowered, and gently pressed onto the thinned area, held in place by a 201 stereotactic arm. After glue solidification (several minutes), the edges of the glass cover 202 slip were sealed off by dental cement. Following the surgical procedure, mice received 203 pain medication, and were allowed to recover for at least 3 days. The „reinforcement“ of 204 the cranial window prevented the skull from growing back making it possible to image 205 the same cortical structures repeatedly without the need of re-thinning the skull. 
207 Experimental timeline. The experimental timeline is displayed in figure $1 \mathrm{~d}$. Following

208 recovery from surgical establishment of a chronic cranial window, mice were

209 acclimatized to the experimenter, until no signs of distress were present (usually $\sim 3$

210 sessions, 30min each). Then, two imaging sessions were carried out to determine the

211 baseline 3 day ('d') synaptic turnover (d1, d4). In between baseline imaging sessions,

212 mice were habituated to the open field maze (d3), and subjected to a first novel object

213 recognition test ${ }^{16}$ (NOR1, d4). Immediately post imaging on day 4, pMIC was initiated.

214 To document synaptic changes occurring during pMIC, a third imaging session was

215 carried out right at the end of pMIC (d5). After at least 24 hours of recovery from pMIC,

216 mice were subjected to a second NOR (NOR2, d7). Three days after pMIC termination,

217 mice were imaged once again to determine their post-anesthetic 3 day synaptic

218 turnover (d8).

219

220 Prolonged medically induced coma. The tightly controlled and safe pMIC procedure

221 (-24hrs) required the continuous presence of an experimenter. Mimicking hospital

222 conditions, remote video and vital parameter monitoring allowed the experimenter to

223 leave the pMIC setup while maintaining the ability to rapidly carry out setup

224 adjustments. Based on a protocol of long-term anesthesia in mice ${ }^{27}$, we established a

225 setup that permits continuous general anesthesia of mice for up to days (Fig. 1a). While

226 anesthetized, mice were kept on a warming pad maintaining a body core temperature of

$22737.5^{\circ} \mathrm{C}$ through a rectal probe and closed-loop temperature control system (ATC2000,

228 World Precision Instruments). The warming pad was placed on a tiltable platform whose

229 angle was slightly changed intermittently $\left(\max .4^{\circ}\right)$ in order to shift the animals center of 
230 mass to reduce pressure pain and bruising. As mice loose body temperature easily due

231 to their anatomy, an infrared lamp was additionally put in place and optionally switched

232 on, if needed. Isoflurane was delivered in humidified air through a custom nose piece

233 (initial dose 1.5-2.5\% partial pressure in air [ppa], steady state dose $0.5-1.5 \%$ ppa).

234 Ventilation air was humidified between the isoflurane vaporizer and the nose piece, and

235 all waste gas was actively removed via a suction pump. By the help of a dual gas

236 flowmeter system $\left(100 \% \quad \mathrm{O}_{2}\right.$, and room air) set up before the isoflurane vaporizer

237 system, the oxygen fraction $\left(\mathrm{FiO}_{2}\right)$ in air could be modified seamlessly between 21-

$238100 \% . \mathrm{FiO}_{2}$ adjustment depended on the measured peripheral arterial $\mathrm{O}_{2}$ saturation.

239 During the early period of pMIC, a fraction of inspired oxygenation $\left(\mathrm{FiO}_{2}\right)$ of $21 \%$ was

240 completely sufficient to maintain high peripheral $\mathrm{O}_{2}$ saturation in all anesthetized mice,

241 while subsequently $(>8 \mathrm{hrs}) \quad \mathrm{FiO}_{2}$ was usually slowly increased towards pMIC

242 termination without exceeding 50\%. Similarly to human intensive care, we tried to avoid

243 an $\mathrm{fiO}_{2}>50 \%^{18}$. To provide the animal with sufficient fluid and nutrition during

244 anesthesia, continuous fluid (saline) and nutritional supply ( $1 \%$ glucose solution, in

245 PBS) was administered via a programmable perfusion system via a subcutaneous line

246 at a rate of $0.1-0.2 \mathrm{ml} / \mathrm{hr}$. All throughout anesthesia, basic vital parameters (blood

247 oxygenation, heart rate, breath rate, pulse distension, breath distension) were

248 continuously monitored and recorded by a thigh or paw sensor (mouse ox plus system,

249 Starr life sciences). The sensor was periodically moved from one extremity to another to

250 avoid pressure-induced tissue damage. Further, this approach minimized the risk of

251 faulty vital parameter sensing due to excessive tissue compression. A sustained

252 pulse/breath distension ratio below 1 was sought to be avoided $^{27}$, as it indicates 
253 anesthesia being too deep (large inhalatory excursions, gasping; in combination with

254 brady- or normocardia), or a lack of intravascular fluid (hypovolemia; in combination with

255 tachycardia). The experimenter was continually present in the laboratory to perform

256 imaging immediately prior and post pMIC, and to adjust for example anesthetic depth or

257 fluid supply with minimal delay all throughout anesthesia. Real-time streaming of video

258 monitoring (Thorlabs, DCC1645C) and vital parameters through a local network allowed

259 for remote monitoring of anesthetized animals.

261 Two-photon imaging. For chronic surveillance of structural synaptic changes in vivo,

262 dendritic arborization, and dendritic spines of layer 5 cortical neurons expressing yellow

263 fluorescent protein (YFP) ${ }^{14}$ were imaged 4 times (day 1, day 4 [start of anesthesia], day

2645 [end of anesthesia], day 8) using a two-photon microscope (Bruker Ultima; Billerica,

$265 \mathrm{MA}$ ) and a Ti:Sapphire laser (Chameleon Ultra II; Coherent) at $940 \mathrm{~nm}$ through a 25x

266 objective (water immersion, N.A. 1,05, Olympus). Galvanometer scanning and image

267 acquisition was controlled by Prairie View Imaging software. Each imaging session

268 ( 15-30 minutes) included 1-4 high resolution z-stacks in different locations (field of

269 view typically $\sim 50 \times 50 \mu \mathrm{m}, 512 \times 512$ pixels, step size $1.5 \mu \mathrm{m}$, stack depth up to 150

$270 \mu \mathrm{m}$ beneath the pial surface). Imaged z-stacks were precisely co-registered across each

271 imaging session by vascular landmarks and several different optical zoom average

272 images of the cortex. For each imaging session, head-restrained animals were kept

273 under light isoflurane anesthesia (0.5-1\% partial pressure in air) via a nose piece while

274 body temperature was maintained with a warming pad $\left(37.5^{\circ} \mathrm{C}\right)$. 
276 Novel object recognition test. Before and after anesthesia, mice were subjected to

277 cognitive testing by use of a novel object recognition test (NOR), a widely used basic

278 measure of cognitive function and recognition memory in mice ${ }^{16}$. Following habituation

279 (exp. day 3 , figure $1 \mathrm{~d})$ to an open field maze $(50 \times 50 \mathrm{~cm})$, mice underwent a first NOR

280 (exp. day 4, prior to start of pMIC). Initially, mice were exposed to two identical objects

281 (e.g. cell culture flasks filled with sand, or LEGO towers) that were placed in the open

282 field for 10 minutes ("familiarization"). After familiarization, mice returned to their home

283 cage for 4-5 hours, upon which they were placed in the open field maze again, yet with

284 one of the two identical objects replaced by a novel object (cell culture flask, or lego

285 tower, respectively). The NOR was repeated with a new set of objects at least 24 hours

286 after pMIC termination (exp. day 7), so changes in basic cognitive function, specifically

287 recognition memory, could be evaluated. All behavioral testing was video-recorded (7.5

$288 \mathrm{fr} / \mathrm{sec}$ ) from above the open field. We used custom written Matlab code for automated

289 tracking (travelled distance, object exploration time) of mouse exploratory behavior

290 during NOR (Fig. 1c).

291

292 Data analysis. Similarly to previously described procedures for quantifying synapse

293 dynamics ${ }^{28}$, stacks were analyzed using the freely available software ImageJ

294 (rsbweb.nih.gov/ij/). Individual 3d-stacks for each time point (d1, d4, d5, d8), and

295 imaged areas (1-4 per animal) were analyzed side-by-side by two examiners who were

296 blinded to conditions, except for the reference imaging timepoint (d1). Based on

297 consensus between the two blinded examiners, clearly visible protrusions on dendritic

298 segments were classified as synapses. For each dendritic segment analyzed, same 
299 synapses were defined as present (1) or absent (0) across stacks (imaging time points).

300 Based on the examiners' consensus, synapses could be defined as stable, gained, or

301 eliminated across imaging time points, once an entire experiment was analyzed, and

302 imaging time points could be revealed, and thus re-assigned to individual stacks.

303 Statistical differences between synaptic turnover, synaptic gain, or loss were tested

304 using paired (pre- vs. post-anesthetic), or unpaired (control group vs. anesthetized

305 group) student's t-test. All statistical analyses were carried out using MATLAB-R2017b

306 (MathWorks), and Prism 5 (GraphPad Software).

307

308 Data availability statement. The data that support the findings of this study are

309 available from the corresponding author upon reasonable request.

311 Code availability statement. The custom MATLAB code for automated behavioral

312 tracking is available at: https://github.com/hanshuting/mnovobj 


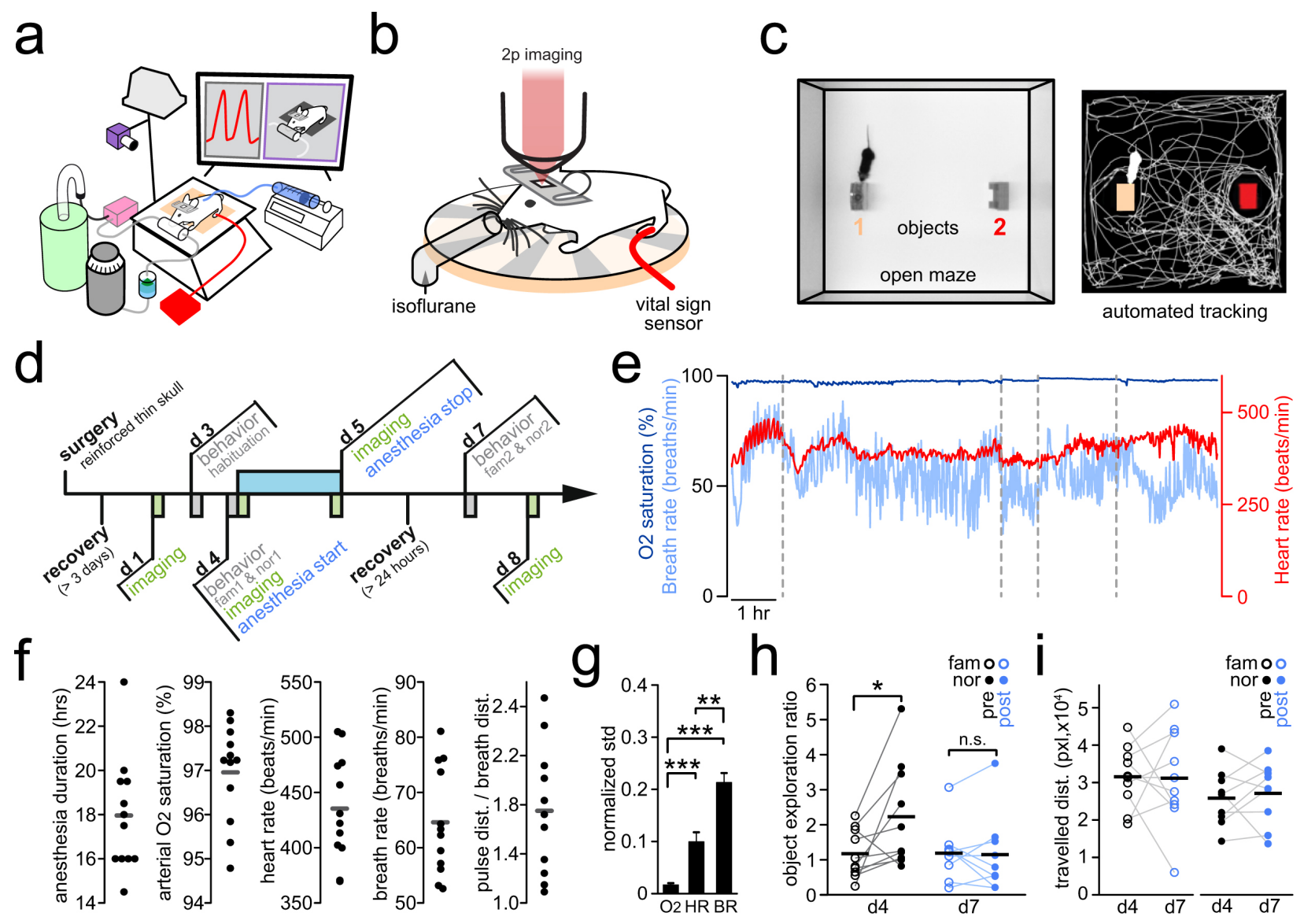

Figure 1. Experimental design and pMIC-induced behavioral deficits

319 a) Sketch of the anesthesia setup for pMIC. The mouse is placed on a warming pad on a tiltable platform

320 with its nose resting in a custom nose piece. Isoflurane is evaporated (grey), and delivered in humidified

321 air/oxygen. Waste gas is removed via a suction pump (magenta) and trapped in a charcoal filter

322 (turquoise). Nutritional supply is delivered via a subcutaneous line by a programmable perfusion system

323 (blue, right). In addition to the warming pad, an infrared lamp is placed above the setup. Vital parameters

324 are measured using a thigh sensor (red). Vital parameters and clinical status are video recorded and live-

325 streamed in a local network (camera above the setup, cmputer display). b) for individual imaging sessions

326 (max. $30 \mathrm{~min}$ ), the mouse is placed under a two-photon microscope, on a warming pad, under isoflurane

327 anesthesia, while vital signs are being monitored using a thigh sensor. c) open maze for behavioral

328 testing. A camera is mounted atop the maze. Videos are recorded during object familiarization and novel

329 object recognition, and the mouse behavior is automatically tracked using custom written MATLAB code.

330 d) Experimental workflow, fam $=$ familiarization, nor $=$ novel object recognition test. e) Basic vital 
331 parameter long-term monitoring of an anesthetized mouse, with intermittent breaks (dotted lines, e.g. for

332 moving the sensor from one extremity to another). Faulty measurements for technical reasons (e.g. tissue

333 compression) are excluded. Displayed recording length $\sim 12$ hrs. f) measured set of vital parameters,

334 mean values ( $n=12$ mice), overall mean value displayed as horizontal grey line. dist. = distension. $\mathbf{g}$ )

335 Comparison of the core vital parameter normalized mean standard deviations $(n=12$ mice $)$. Unpaired $t$ -

336 test with Welsh's correction: $\mathrm{O} 2$ saturation vs. heart rate $\mathrm{p}=0.001, \mathrm{O} 2$ saturation vs. breath rate $\mathrm{p}<0.0001$,

337 heart rate vs. breath rate $p=0.0002$. h) basic cognitive testing: relative object exploration time ratio ( 2

338 objects, $50 \%$ vs. $50 \%=1$ ) during familiarization ("fam", circles, 2 identical objects), followed by novel

339 object recognition ("nor", filled circles, 1 of the identical objects replaced by a novel object) measured

340 during baseline (d4), and post recovery from pMIC (d7). Paired t-test: fam (d4) vs. nor (d4) p=0.0451

341 ( $n=10$ mice), fam (d7) vs. nor (d7) $p=0.823$ ( $n=9$ mice). i) the reduction in "nor" performance can not be

342 explained by reduced locomotion, travelled distances (in pixels) prior and post pMIC during fam (circles),

343 and nor (filled circles), are consistent. Depiction of statistical significance in fig. $1:{ }^{*} p<0.05,{ }^{* *} p<0.01$,

$344{ }^{* * *} p<0.001$. All bar plots show mean \pm s.e.m.. 

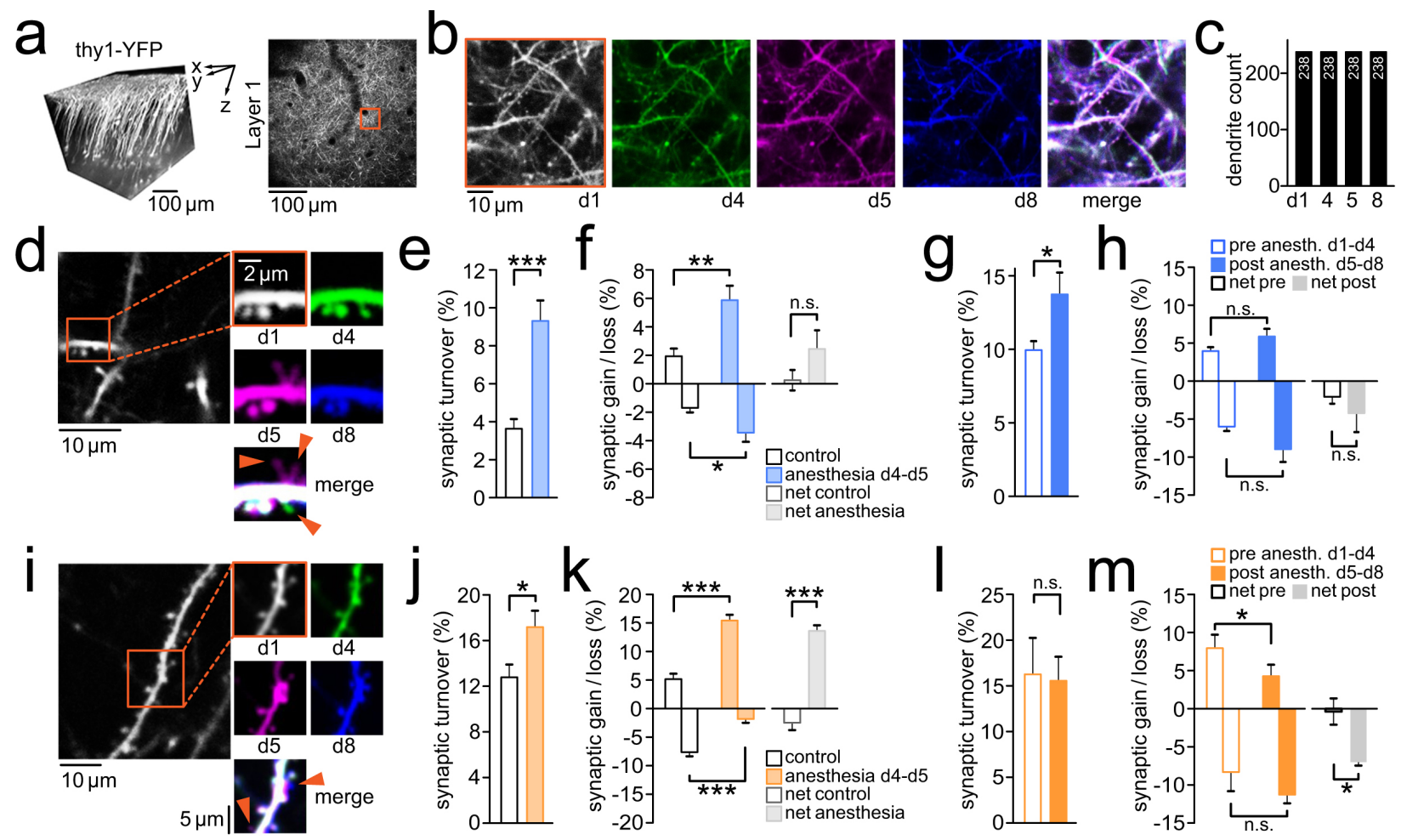

Figure 2. pMIC leads to synaptic alterations in adolescence and adulthood

a) Left: 3D reconstruction of imaged layer 5 pyramidal neurons in vivo. Imaging was typically carried out up until $150 \mu m$ beneath the pial surface. Right: Axial imaging field of view (FOV) at low magnification just below the brain surface to precisely re-identify highly zoomed-in FOVs across multiple imaging sessions using vascular landmarks. b) red inset shown in a; identical dendritic segments were imaged 4 times before pMIC (d1, d4), and following pMIC $(\mathrm{d} 5, \mathrm{~d} 8)$ in an adult mouse, with a complete overlap of dendritic segments across imaging sessions (right). c) Quantification of structural dendritic changes across pMIC in adult mice $(n=6)$; dendritic segments remained completely stable, with no dendrites gained, or lost. d) Paradigmatic dendritic segment with associated synaptic changes across pMIC (baseline: d1-d4, pMIC: d4-d5, post pMIC: d5-d8). Two synapses are transiently gained during pMIC (magenta), 1 synapse is permanently lost (green). e) Total synaptic turnover during pMIC in adult mice ( $n=6)$ vs. 1-day turnover in healthy controls $(n=6)$; unpaired t-test: $p=0.043$ f) Synaptic gain, synaptic loss, and net-effect (gain - loss)

360 during pMIC in adult mice $(n=6)$ vs. healthy controls $(n=6)$; unpaired t-test: $p=0.0064$ (gain), $p=0.0354$

361 (loss), p=0.1728 (net-effect). g) Total 3-day synaptic turnover within adult mice $(n=6)$ during baseline (d1d4) vs. post pMIC (d5-d8); paired t-test: $p=0.0484$ h) Synaptic gain, synaptic loss, and net-effect (gain - 
363 loss) within adult mice $(n=6)$ during baseline (d1-d4) vs. post pMIC (d5-d8); paired t-test:p=0.4913 (gain), $364 p=0.16$ (loss), $p=0.4441$ (net-effect). i) Paradigmatic dendritic segment with associated synaptic changes 365 across pMIC (baseline: d1-d4, pMIC: d4-d5, post pMIC: d5-d8). Two synapses are gained during pMIC 366 (magenta), and persist during the post pMIC period (blue). j) Total synaptic turnover during pMIC in 367 adolescent mice $(n=4)$ vs. 1-day turnover in healthy controls $(n=5)$; unpaired t-test: $p=0.0433$ k) Synaptic 368 gain, synaptic loss, and net-effect (gain - loss) during pMIC in adolescent mice $(n=4)$ vs. healthy controls $369(n=5)$; unpaired t-test: $p=0.0002$ (gain), $p=0.0007$ (loss), $p<0.0001$ (net-effect). I) Total 3-day synaptic 370 turnover within adolescent mice $(n=4)$ during baseline $(d 1-d 4)$ vs. post pMIC (d5-d8); paired t-test: $371 p=0.7099 \mathrm{~m}$ ) Synaptic gain, synaptic loss, and net-effect (gain - loss) within adolescent mice $(\mathrm{n}=4)$ during 372 baseline (d1-d4) vs. post pMIC (d5-d8); paired t-test: $p=0.043$ (gain), $p=0.1671$ (loss), $p=0.0386$ (net373 effect). Depiction of statistical significance in fig. 2 : ${ }^{*} p<0.05$, ${ }^{* *} p<0.01$, ${ }^{* *} p<0.001$. All bar plots show 374 mean \pm s.e.m.

375 
389 1. Pandharipande, P. P. et al. Long-Term Cognitive Impairment after Critical Illness. N. Engl. J. Med.

$390 \quad 369,1306-1316(2013)$.

391 2. Cox, C. E. \& Carson, S. S. Medical and economic implications of prolonged mechanical ventilation

392 and expedited post-acute care. Semin. Respir. Crit. Care Med. (2012). doi:10.1055/s-0032-

$393 \quad 1321985$

394 3. Hopkins, R. O. \& Girard, T. D. Medical and economic implications of cognitive and psychiatric 395 disability of survivorship. Semin. Respir. Crit. Care Med. (2012). doi:10.1055/s-0032-1321984

396 4. Norman, B. C. et al. Employment Outcomes after Critical Illness: An Analysis of the Bringing to 397 Light the Risk Factors and Incidence of Neuropsychological Dysfunction in ICU Survivors Cohort. 398 Crit. Care Med. (2016). doi:10.1097/CCM.0000000000001849

399 5. Unroe, M. et al. One-year trajectories of care and resource utilization for recipients of prolonged 400 mechanical ventilation: A cohort study. Ann. Intern. Med. (2010). doi:10.7326/0003-4819-153-3-

$401 \quad 201008030-00007$

402 6. Kohler, J. et al. Cognitive deficits following intensive care. Dtsch Arztebl Int 116, 627-634 (2019).

403 7. Briner, A. et al. Developmental stage-dependent persistent impact of propofol anesthesia on 404 dendritic spines in the rat medial prefrontal cortex. Anesthesiology (2011).

405 doi:10.1097/ALN.0b013e318221fbbd

406 8. De Roo, M. et al. Anesthetics rapidly promote synaptogenesis during a critical period of brain 407 development. PLoS One (2009). doi:10.1371/journal.pone.0007043

408 9. Sanders, R. D., Hassell, J., Davidson, A. J., Robertson, N. J. \& Ma, D. Impact of anaesthetics and 409 surgery on neurodevelopment: an update. Br J Anaesth 110 Suppl, i53-72 (2013).

410 10. Grutzendler, J., Kasthuri, N. \& Gan, W. B. Long-term dendritic spine stability in the adult cortex. $411 \quad$ Nature (2002). doi:10.1038/nature01276

412 11. Zuo, Y., Lin, A., Chang, P. \& Gan, W. B. Development of long-term dendritic spine stability in 413 diverse regions of cerebral cortex. Neuron 46, 181-189 (2005).

414 12. Trachtenberg, J. T. et al. Long-term in vivo imaging of experience-dependent synaptic plasticity in 415 adult cortex. Nature (2002). doi:10.1038/nature01273 
416 13. Yang, G., Chang, P. C., Bekker, A., Blanck, T. J. J. \& Gan, W. B. Transient effects of anesthetics on dendritic spines and filopodia in the living mouse cortex. Anesthesiology 115, 718-726 (2011).

418 14. Feng, G. et al. Imaging neuronal subsets in transgenic mice expressing multiple spectral variants of GFP. Neuron (2000). doi:10.1016/S0896-6273(00)00084-2

420 15. Drew, P. J. et al. Chronic optical access through a polished and reinforced thinned skull. Nat.

$421 \quad$ Methods (2010). doi:10.1038/nmeth.1530

422 16. Leger, M. et al. Object recognition test in mice. Nat. Protoc. (2013). doi:10.1038/nprot.2013.155

423 17. Yasuda, N. et al. Kinetics of desflurane, isoflurane, and halothane in humans. Anesthesiology (1991). doi:10.1097/00000542-199103000-00017

425 18. de Jonge, E. et al. Association between administered oxygen, arterial partial oxygen pressure and mortality in mechanically ventilated intensive care unit patients. Crit. Care (2008). doi:10.1186/cc7150

428 19. Segal, M. Dendritic spines: Morphological building blocks of memory. Neurobiol. Learn. Mem. 138, $429 \quad 3-9(2017)$.

430 20. Yuste, R. Dendritic spines and distributed circuits. Neuron 71, 772-781 (2011).

431 21. Fu, M. \& Zuo, Y. Experience-dependent structural plasticity in the cortex. Trends Neurosci. 34, 432 177-187 (2011).

433 22. Århem, P., Klement, G. \& Nilsson, J. Mechanisms of anesthesia: Towards integrating network, $434 \quad$ cellular, and molecular level modeling. Neuropsychopharmacology (2003).

435 doi:10.1038/sj.npp.1300142

436 23. Krasowski, M. D. \& Harrison, N. L. General anaesthetic actions on ligand-gated ion channels. 437 Cellular and Molecular Life Sciences (1999). doi:10.1007/s000180050371

438 24. Kaech, S., Brinkhaus, H. \& Matus, A. Volatile anesthetics block actin-based motility in dendritic spines. Proc. Natl. Acad. Sci. U. S. A. 96, 10433-10437 (1999).

44025 Liston, C. et al. Circadian glucocorticoid oscillations promote learning-dependent synapse 441 formation and maintenance. Nat. Neurosci. 16, 698-705 (2013).

442 26. Xu, H. T., Pan, F., Yang, G. \& Gan, W. B. Choice of cranial window type for in vivo imaging affects 443 dendritic spine turnover in the cortex. Nat. Neurosci. 10, 549-551 (2007). 
444 27. Ewald, A. J., Werb, Z. \& Egeblad, M. Monitoring of vital signs for long-term survival of mice under 445 anesthesia. Cold Spring Harb Protoc 2011, pdb prot5563 (2011).

446 28. Yang, G., Pan, F. \& Gan, W. B. Stably maintained dendritic spines are associated with lifelong 447 memories. Nature 462, 920-924 (2009). 